\title{
Single branch of chiral Majorana modes from doubly degenerate Fermi surfaces
}

\author{
Wang Yang, Chao Xu ${ }^{\circledR}$, and Congjun Wu ${ }^{\circ}$ \\ Department of Physics, University of California, San Diego, California 92093, USA
}

(Received 16 December 2019; revised 14 November 2020; accepted 24 November 2020; published 24 December 2020)

\begin{abstract}
Majorana fermions are often proposed to be realized by singling out one Fermi surface without spin degeneracy via spin-orbit coupling, and then imposing boundaries or defects. In this work, we take a different route starting with two degenerate Fermi surfaces without spin-orbit coupling, and show that by the method of "kink on boundary," the dispersive chiral Majorana fermions can be realized in superconducting systems with $p \pm i s$ pairings. The surfaces of these systems develop spontaneous magnetizations whose directions are determined by the boundary orientations and the phase difference between the $p$ - and $s$-component gap functions. Along the magnetic domain walls on the surface, there exist chiral Majorana fermions propagating unidirectionally, which can be conveniently dragged and controlled by external magnetic fields. Furthermore, the surface magnetization is shown to be a magnetoelectric effect based on a Ginzburg-Landau free-energy analysis. We also discuss how to use the proximity effects to realize chiral Majorana fermions by performing the kink on boundary method.
\end{abstract}

DOI: 10.1103/PhysRevResearch.2.042047

Majorana fermions are their own antiparticles which were first introduced to high-energy physics [1]. In the past decade, they have been intensively investigated in the context of condensed matter physics [2]. The braiding of Majorana particles exhibits non-Abelian statistics [3-6], which is distinct from the usual Fermi and Bose statistics and can be applied for quantum computations [7-11]. The topological nature of Majorana modes makes the braiding and fusion operations robust from the decoherence processes which are detrimental to the realization of quantum computers.

The Majorana modes are proposed to exist in the $v=$ $\frac{5}{2}$ fractional quantum Hall state [3]. There have also been considerable interests in studying Majorana fermions in topological superconducting systems [12-17]. Majorana fermions appear on boundaries, in vortex cores, and at defects of topological superconducting systems [5,18-23]. The chiral Majorana fermion has been proposed to emerge in the quantum anomalous Hall insulator in proximity with an $s$-wave superconductor [24-26]. The interaction effects in Majorana fermions have also been discussed by various authors [27-29]. Recent experiments have provided evidence to the existence of Majorana zero modes and chiral Majorana fermions in condensed matter systems [30-32].

A Majorana fermion is half of a usual fermion in view of the degrees of freedom that it contains. Since electrons have two spin degrees of freedom, the chiral Majorana fermions can only be obtained by a "half of half" method. Typically, the first "half" is achieved by singling out a nondegenerate Fermi surface in spin-orbit coupled systems, which becomes

Published by the American Physical Society under the terms of the Creative Commons Attribution 4.0 International license. Further distribution of this work must maintain attribution to the author(s) and the published article's title, journal citation, and DOI. effectively single component. The second "half" is performed by imposing boundaries or defects to generate zero modes.

On the other hand, there has been considerable experimental and theoretical interest in studying superconducting states with competing singlet and triplet pairings [33-43]. A spontaneously time-reversal symmetry breaking mixing is energetically favored exhibiting $\pm \frac{\pi}{2}$ phase difference between the gap functions in these two different channels [34]. This class of novel pairing states has been proposed in the ultracold electric dipolar fermion systems [34], in cold fermion systems under $p$-wave Feshbach resonances [42], in iron-pnicitide superconductors [41], and in inversion symmetry breaking superconducting systems [43]. Such kind of time-reversal symmetry breaking superconductors host gapped Dirac cones on the surface with nontrivial gravitational responses and thermal Hall effects [43-49]. Recently, there has been strong experimental evidence for unconventional and time-reversal symmetry breaking pairing in the superconducting state of the noncentrosymmetric material $\mathrm{Re}_{0.82} \mathrm{Nb}_{0.18}$ [50], which is very likely of a mixed singlet and triplet nature [51].

In this Rapid Commnication, we analyze the formation of chiral Majorana fermions in superconductors with mixed singlet and triplet pairings of the $p \pm i$ s type. Different from previous works, there are two degenerate Fermi surfaces without any spin-orbit coupling, and the strategy of "half of half" is implemented as "kink on boundary." The boundaries of the $p \pm i s$ superconductors are shown to be spontaneously spin polarized, and the magnetizations are opposite for $p+i s$ and $p-i s$ pairings. As a result, the "kink" formed by the domain wall between the $p+i s$ and $p-i$ s superconducting regions on the surface is also a magnetization domain wall. We show that there exists a chiral Majorana fermion propagating unidirectionally along the "kink." The spirit of such kink on boundary method is similar to realizing Majorana corner and hinge modes in the high-order topological systems [52-59]. However, the chiral Majorana fermions realized using our 
method are mobile in the sense that they can be dragged and controlled by external magnetic fields, hence convenient for braiding purposes. In addition, we show that the spontaneous surface magnetization is a manifestation of a novel magnetoelectric effect based on a Ginzburg-Landau free-energy analysis. Finally, we also discuss how to use the proximity effects in triplet superconductors including $\mathrm{Cu}_{x} \mathrm{Bi}_{2} \mathrm{Se}_{3}$ and $\mathrm{Sn}_{1-x} \mathrm{In}_{x}$ Te to realize chiral Majorana fermions by performing the kink on boundary method.

We consider a gap function structure with a dominant $p$-wave component mixed with an $s$-wave one. It typically prefers the time-reversal symmetry breaking pairing pattern $p \pm i s$ [34]. The corresponding gap function matrix reads $\Delta_{\alpha \beta}(\vec{k})=\Delta_{s}(\vec{k})\left(i \sigma_{2}\right)_{\alpha \beta}+\Delta_{p}(\vec{k}) \hat{d}(\vec{k}) \cdot\left(i \vec{\sigma} \sigma_{2}\right)_{\alpha \beta}$ where $\hat{d}(\vec{k})$ is a unit real vector and $\vec{\sigma}$ 's are the Pauli matrices in spin space. Only when the phase difference between $\Delta_{s}$ and $\Delta_{p}$ equals to $\pm \frac{\pi}{2}$, is $\Delta_{\alpha \beta}$ proportional to a unitary matrix. Typically, unitary pairings are energetically more favorable over nonunitary ones [60]. To see this, consider the following Ginzburg-Landau free energy

$$
\begin{aligned}
F= & -\alpha_{s} \Delta_{s}^{*} \Delta_{s}-\alpha_{p} \Delta_{p}^{*} \Delta_{p}+\beta_{s}\left|\Delta_{s}\right|^{4}+\beta_{p}\left|\Delta_{p}\right|^{4} \\
& +\gamma_{1}\left|\Delta_{p}\right|^{2}\left|\Delta_{s}\right|^{2}+\gamma_{2}\left(\Delta_{p}^{*} \Delta_{p}^{*} \Delta_{s} \Delta_{s}+\text { c.c. }\right),
\end{aligned}
$$

in which both $\alpha_{s}$ and $\alpha_{p}$ are negative, signaling instabilities in both $s$ - and $p$-wave channels. Equation (1) is the most general form of the free energy up to quartic order which respects time-reversal and inversion symmetries. Since $\gamma_{2}$ is positive generically [43], a $\pm \pi / 2$ phase difference between $\Delta_{s}$ and $\Delta_{p}$ is energetically favored. Up to an overall gauge transformation, the $p$ - and $s$-wave components can be fixed as real and imaginary, respectively. Both time-reversal $(\mathcal{T})$ and inversion $(\mathcal{P})$ symmetries are spontaneously broken in the $p \pm i$ is pairing states. Nevertheless, the system is invariant up to an overall phase under the $\mathcal{P} \mathcal{T}$ transformation, i.e., the combined parity and time-reversal operations.

For simplicity, we start with a 1D $p_{z} \pm i$ s superconductor, whose Bogoliubov-de Gennes (BdG) Hamiltonian reads

$$
\begin{aligned}
H_{1 \mathrm{D}}= & \frac{1}{2} \int d z \psi^{\dagger}(z)\left\{\left[-\frac{\hbar^{2}}{2 m} \partial_{z}^{2}-\mu(z)\right] \tau_{3}-\Delta_{s} \sigma_{2} \tau_{1}\right. \\
& \left.-\frac{\Delta_{p}}{k_{f}} i \partial_{z} \sigma_{1} \tau_{1}\right\} \psi(z),
\end{aligned}
$$

in which $\psi(z)=\left[c_{\uparrow}^{\dagger}(z) c_{\downarrow}^{\dagger}(z) c_{\uparrow}(z) c_{\downarrow}(z)\right]^{T} ; \tau_{i}$ 's are the Pauli matrices in the Nambu space; $k_{f}$ is the Fermi wave vector; and $\Delta_{s}$ and $\Delta_{p}$ represent the singlet and triplet pairing gap functions which are assumed real without loss of generality. The BdG Hamiltonian, Eq. (2), possesses the particle-hole symmetry $P_{h} H_{1 \mathrm{D}} P_{h}^{-1}=-H_{1 \mathrm{D}}$ where $P_{h}$ is an antiunitary transformation defined as $P_{h} \psi^{\dagger}(z) P_{h}^{-1}=\psi^{\dagger}(z) \sigma_{0} \tau_{1} K$, with $K$ the complex conjugate operation. The triplet pairing pattern in Eq. (2) corresponds to the $d$-vector configuration $\hat{d} \| \hat{z}$, hence the $z$ component of spin is conserved, represented as $S_{z}=\frac{1}{4} \sigma_{3} \tau_{3}$. In the absence of $\Delta_{s}$, the system preserves timereversal symmetry $T \psi^{\dagger}(z) T^{-1}=\psi^{\dagger}(z) i \sigma_{2} \tau_{0} K$, and there exists a chiral operator $C_{\mathrm{ch}}=-i T P_{h}=\sigma_{2} \tau_{1}$ anticommuting with the Hamiltonian. The chiral operator in general maps positive energy states to negative energy states, but becomes a symmetry for the zero modes. In what follows, an open boundary condition is imposed along the $z$ direction at the upper $\left(z=\frac{L}{2}\right)$ and lower $\left(z=-\frac{L}{2}\right)$ edges, with $\mu(z)=\frac{\hbar^{2}}{2 m} k_{f}^{2}$ at $|z|<\frac{L}{2}$ and $\mu(z)=-\infty$ at $|z|>\frac{L}{2}$, where $L$ is the system size.

When $\Delta_{s}=0$, there exist two Majorana zero modes at each edge of the system. The associated creation operators for the four Majorana modes are $\gamma_{\lambda}^{a, \dagger}=\int d z \psi^{\dagger}(z) \Psi_{\lambda}^{a}(z)$, in which $a=+(-)$ for upper (lower) edge and $\lambda= \pm$ labeling the two zero modes at each edge. The zero mode wave functions $\Psi_{\lambda}^{a}$ are solved as $\Psi_{+}^{a}(z)=\frac{1}{\sqrt{2}}\left(e^{-i a(\pi / 4)}, 0,0, e^{i a(\pi / 4)}\right)^{T} u_{+}(z)$ and $\Psi_{-}^{a}(z)=\frac{1}{\sqrt{2}}\left(0, e^{-i a(\pi / 4)}, e^{i a(\pi / 4)}, 0\right)^{T} u_{-}(z), \quad$ respectively, where $u_{a}(z)$ is the envelope function with the expression given in Supplemental Material (SM) Sec. I [61]. Since $\left[C_{\mathrm{ch}}, S_{z}\right]=0$, the wave functions of the four zero modes can be chosen as the simultaneous eigenstates of $C_{\mathrm{ch}}$ and $S_{z}$ :

$$
C_{\mathrm{ch}} \Psi_{\lambda}^{a}=a \lambda \Psi_{\lambda}^{a}, S_{z} \Psi_{\lambda}^{a}=\frac{1}{2} \lambda \Psi_{\lambda}^{a} .
$$

Furthermore, there exists an emergent supersymmetry expressed as $\gamma_{+}^{a}=\gamma_{-}^{a, \dagger}[62]$.

When $\Delta_{s} \neq 0$, the four modes become gapped, and a spontaneous magnetization develops on the edge. Since the singlet pairing component in $H_{1 \mathrm{D}}$ is $-\Delta_{s} C_{\mathrm{ch}}, C_{\mathrm{ch}}$ and $S_{z}$ still form a complete set of good quantum numbers for the four modes as expressed in Eq. (3). Without loss of generality, let us consider the case of $\Delta_{s}>0 . \gamma_{\lambda=a}^{a, \dagger}$ represents the quasiparticle annihilation operator, since $\left[H_{1}, \gamma_{\lambda=a}^{a, \dagger}\right]=-\Delta_{s} \gamma_{\lambda=a}^{a, \dagger}$. The projection of the $S_{z}$ operator to the edge state subspace can be expressed as $S_{z}=-\frac{1}{2} a\left(\gamma_{\lambda=-a}^{a, \dagger} \gamma_{\lambda=a}^{a, \dagger}-\frac{1}{2}\right)$, hence,

$$
\left\langle G\left|S_{z}\right| G\right\rangle=\frac{1}{4} a,
$$

where $a= \pm 1$, and $|G\rangle$ is the ground state of the system. This result shows the fractionalization of the $s_{z}$ eigenvalue to $\pm \frac{1}{4}$ on the boundary. Therefore, the upper and lower edges carry spontaneous magnetizations along the $z$ direction, and they are oppositely magnetized as enforced by the $\mathcal{P} \mathcal{T}$ symmetry. Define the $\mathcal{P} \mathcal{T}$ operation as $\mathcal{S}=G \mathcal{P} \mathcal{T}$, where $G: c_{\sigma}(z) \rightarrow$ $i c_{\sigma}(z)(\sigma=\uparrow, \downarrow)$ is a gauge transformation. The operation $\mathcal{S}$ flips both $a$ and $\lambda$ and maintains the $C_{\mathrm{ch}}$ index invariant, since $\mathcal{S}$ switches the upper and lower edges, $\left\{\mathcal{S}, S_{z}\right\}=0$, and $\left[\mathcal{S}, C_{\mathrm{ch}}\right]=0$. As a result, $\gamma_{\lambda=a}^{a, \dagger}$ and $\gamma_{\lambda=-a}^{-a, \dagger}$ are related by $\mathcal{S}$ and are the eigenoperators with the same energy eigenvalue. Hence, the magnetizations are opposite for the two edges. As for the case of $\Delta_{s}<0$, the magnetization at each edge is reversed with respect to the case of $\Delta_{s}>0$. We also note that there is no magnetization in the bulk due to the $\mathcal{P} \mathcal{T}$ symmetry.

Next we consider the $p \pm i$ s superconductors in two dimensions and show that there appears a single Majorana zero mode localized at the magnetic kink on the 1D edge of this system. The corresponding BdG Hamiltonian reads

$$
\begin{aligned}
H_{2 \mathrm{D}}= & \frac{1}{2} \int d^{2} \vec{r} \psi^{\dagger}(\vec{r})\left\{\left[-\frac{\hbar^{2}}{2 m}\left(\partial_{y}^{2}+\partial_{z}^{2}\right)-\mu(z)\right] \tau_{3}\right. \\
& \left.-\Delta_{s}(y) \sigma_{2} \tau_{1}+\frac{1}{k_{f}}\left(\Delta_{p}^{y} i \partial_{y} \tau_{2}-\Delta_{p}^{z} i \partial_{z} \sigma_{1} \tau_{1}\right)\right\} \psi(\vec{r}),
\end{aligned}
$$


in which $\vec{r}=(y, z), \Delta_{p}^{y, z}$ represent the triplet pairing strengths in the $p_{y}, p_{z}$ partial wave channels, respectively, and the $d$ vector is pointing along the momentum direction. Again an open boundary condition is imposed along the $z$ direction. With a uniform $\Delta_{s}(y)$, the momentum $k_{y}$ along the $y$ direction is a good quantum number. The Hamiltonian in Eq. (5) reduces back to Eq. (2) by setting $k_{y}=0$, thus there are two gapped modes $\Psi_{ \pm}^{a}$ on each edge $a$.

At a small but nonzero $k_{y}$, the effective 1D low-energy edge Hamiltonian can be obtained by the $\boldsymbol{k} \cdot \boldsymbol{p}$ method, as $H_{2 \mathrm{D} \text {, edge }}^{a}=-a\left(\Delta_{s} s_{3}+\frac{\Delta_{p}^{y}}{k_{f}} k_{y} s_{1}\right)$, in which $s_{i}$ 's are the Pauli matrices in the basis of $\Psi_{ \pm}^{a}$ for the $a$ edge. For a spatially slowly varying $\Delta_{s}(y)$, the edge Hamiltonian becomes

$$
H_{2 \mathrm{D}, \text { edge }}^{a}=-a\left[\Delta_{s}(y) s_{3}-i \frac{\Delta_{p}^{y}}{k_{f}} \partial_{y} s_{1}\right] .
$$

Since the direction of the edge magnetization is determined by the sign of $\Delta_{s}(y)$, the position where $\Delta_{s}(y)$ changes sign forms a magnetic kink separating regions of opposite directions of magnetizations. Alternatively, $\Delta_{s}(y)$ can be viewed as the mass of the $1 \mathrm{D}$ superconducting spinless fermion model, therefore, a Majorana zero mode emerges at the magnetic kink $[63,64]$. The Majorana zero mode can be solved based on the low-energy edge Hamiltonian in Eq. (6). For a kink with $\operatorname{sgn}\left[\Delta_{s}(y)\right]=-\lambda \operatorname{sgn}(y)$ where $\lambda= \pm$, the wave function of the zero mode at $a$ edge is $W_{\lambda}^{a}(y, z)=$ $\frac{1}{\sqrt{2}}\left[e^{i \lambda(\pi / 4)} \Psi_{+}^{a}(z)+e^{-i \lambda(\pi / 4)} \Psi_{-}^{a}(z)\right] w_{\lambda}(y)$, and the envelope function reads $w_{\lambda}(y)=\frac{1}{N} e^{\lambda \int_{0}^{y} d y^{\prime} k_{f}\left[\Delta_{s}\left(y^{\prime}\right) / \Delta_{p}\right]}$ with $N$ a normalization factor. We have also numerically verified the existence of Majorana zero modes using a lattice model with details included in SM Sec. II [61].

The symmetry properties of the four zero modes $W_{\lambda}^{a}$ 's are analyzed as follows. With the presence of $\Delta_{s}, C_{\mathrm{ch}}$ is no longer a symmetry of the zero modes; nevertheless, a new chiral operator can be chosen as $C_{\mathrm{ch}}^{\prime}=-\sigma_{3} \tau_{1}$ which anticommutes with $H_{2 \mathrm{D}}$ and is a symmetry for $W_{\lambda}^{a}$ 's. When $\Delta_{s}(y)$ is an odd function of $y$, the system is invariant under the operation $M_{y}^{\prime}=G M_{y}$, where the reflection operation $M_{y}$ is defined as $M_{y} \psi^{\dagger}(y, z) M_{y}^{-1}=\psi^{\dagger}(-y, z) i \sigma_{2} \tau_{0}$. Furthermore, $C_{\mathrm{ch}}^{\prime}$ and $M_{y}^{\prime}$ commute and form a complete set of good quantum numbers for the four zero modes $W_{\lambda}^{a}$ 's:

$$
C_{\mathrm{ch}}^{\prime} W_{\lambda}^{a}=-a \lambda W_{\lambda}^{a}, M_{y}^{\prime} W_{\lambda}^{a}=-\lambda W_{\lambda}^{a} .
$$

Note that for a fixed $\lambda$, the $C_{\text {ch }}^{\prime}$ indices of the pair of states $W_{\lambda}^{ \pm}$are opposite while the $M_{y}^{\prime}$ eigenvalues are the same, and for a fixed $a$, both the $C_{\mathrm{ch}}^{\prime}$ indices and the $M_{y}^{\prime}$ eigenvalues of the states $W_{ \pm}^{a}$ are opposite. These are consequences of the symmetries of the system as discussed in SM Sec. III [61].

Now we extend the above discussions to $3 \mathrm{D} p \pm i$ s superconductors, with the following BdG Hamiltonian:

$$
\begin{aligned}
H_{3 \mathrm{D}}= & \frac{1}{2} \int d \vec{r} \psi^{\dagger}(\vec{r})\left\{\left[-\frac{\hbar^{2}}{2 m} \nabla^{2}-\mu(z)\right] \tau_{3}-\Delta_{s}(y) \sigma_{2} \tau_{1}\right. \\
& \left.+\frac{1}{k_{f}}\left(\Delta_{p}^{x} i \partial_{x} \sigma_{3} \tau_{1}+\Delta_{p}^{y} i \partial_{y} \tau_{2}-\Delta_{p}^{z} i \partial_{z} \sigma_{1} \tau_{1}\right)\right\} \psi(\vec{r}),
\end{aligned}
$$

in which $\hat{d}(\vec{k})$ is assumed to be $\hat{k}$. The open boundary condition is imposed along the $z$ direction, the same as before. $H_{3 \mathrm{D}}$ reduces to the Hamiltonian in Eq. (2) when $k_{x}=k_{y}=0$, and to Eq. (5) when $k_{x}=0$.

When $\Delta_{s}(y)=\Delta_{s}$ is a constant, the surface is uniformly spin polarized. The surface modes at $k_{x}=k_{y}=0$ are $\Psi_{\lambda}^{a}$ 's as given in Eq. (3). Away from the surface $\Gamma$ point, the low-energy surface Hamiltonian for the $a$ boundary can be obtained by the $\boldsymbol{k} \cdot \boldsymbol{p}$ method as

$$
H_{\text {surf }}^{a}=a\left[-\Delta_{s} \xi_{3}+\frac{1}{k_{f}}\left(\Delta_{p}^{x} k_{x} \xi_{2}-\Delta_{p}^{y} k_{y} \xi_{1}\right)\right],
$$

where $\xi_{i}$ 's are the Pauli matrices in the basis of $\Psi_{ \pm}^{a}$. For the case of rotationally invariant triplet pairing, i.e., $\Delta_{p}^{j}=\Delta_{p}$ $(j=x, y, z)$, the surface magnetization per unit area is evaluated as $a \frac{k_{f}^{2}}{8 \pi} r\left(\sqrt{1+r^{2}}-r\right)$ where $r=\Delta_{s} / \Delta_{p}$, with detailed calculations included in SM Sec. IV [61]. Due to the surface magnetization, the spontaneous time-reversal symmetry breaking pattern (i.e., the sign of $\Delta_{s}$ ) can be controlled by an external magnetic field. By applying an arbitrarily small field along the positive (negative) $z$ direction to the upper boundary of the system above the superconducting transition temperature, the $p+i s(p-i s)$ state will be favored near the upper boundary when the system is cooled down to be superconducting. The induced symmetry breaking pattern is the opposite for the lower boundary with the same direction of the external field.

There exists a chiral Majorana fermion propagating along the magnetization domain wall where $\Delta_{s}(y)$ changes sign on the boundary of the system. Assuming $\operatorname{sgn}\left[\Delta_{s}(y)\right]=$ $-\lambda \operatorname{sgn}(y)$ with $\lambda= \pm 1$, then for a fixed $\lambda$, there exists a Majorana zero mode $W_{\lambda}^{a}$ with $k_{x}=0$ on the $a$ boundary. In this case, a new chiral operator $C_{\mathrm{ch}}^{\prime}$ which anticommutes with $H_{3 \mathrm{D}}$ (see SM Sec. III [61]) can be defined as $C_{\mathrm{ch}}^{\prime}=G M_{x} T P_{h}$, satisfying $C_{\mathrm{ch}}^{\prime} \psi^{\dagger}(x, y, z) C_{\mathrm{ch}}^{\prime-1}=\psi^{\dagger}(-x, y, z)\left(-\sigma_{3} \tau_{1}\right)$ where $M_{x}$ is the reflection operation defined as $M_{x} \psi^{\dagger}(x, y, z) M_{x}^{-1}=$ $\psi^{\dagger}(-x, y, z) i \sigma_{3} \tau_{1}$. Since $C_{\mathrm{ch}}^{\prime}$ reduces to $-\sigma_{3} \tau_{1}$ when $k_{x}=0$, $C_{\mathrm{ch}}^{\prime}$ is a symmetry for the zero modes. When $k_{x}$ deviates from 0 , the dispersion can be obtained by applying the $\boldsymbol{k} \cdot \boldsymbol{p}$ method to $\Delta H_{\mathrm{ch}}\left(k_{x}\right)=-\frac{\Delta_{p}^{x}}{k_{f}} k_{x} \sigma_{3} \tau_{1}$, which is just $\frac{\Delta_{p}^{x}}{k_{f}} C_{\mathrm{ch}}^{\prime} k_{x}$. Hence, the propagation direction, i.e., the chirality, is determined by the $C_{\mathrm{ch}}^{\prime}$ index and the velocity is $v=C_{\mathrm{ch}}^{\prime} \frac{\Delta_{p}^{x}}{\hbar k_{f}}$. The above analysis is confirmed by numerical computations on a finite size lattice system discussed in SM Sec. II [61]. A schematic plot of the propagation of the Majorana modes is shown in Fig. 1.

We also show that the spontaneous surface magnetization is a manifestation of a magnetoelectric effect. In $p \pm i s$ superconductors, spatial inhomogeneities, including the spatial variations of the external potential $V(\vec{r})$ and the gap functions of the $s$ - and $p$-wave pairings, can all induce spin polarizations. In the following, a Ginzburg-Landau free-energy analysis is presented for the mechanism of the magnetization, which holds for temperatures close to the transition temperature $T_{c}$ and the slow varying case. Denote the magnetic field as $\vec{h}(\vec{r})$, and the singlet and triplet pairing complex gap functions as $\Delta_{s}(\vec{r})$ and $\Delta_{p}(\vec{r})$, respectively, where an isotropic $p$-wave pairing is assumed, i.e., $\Delta_{p}^{j}=\Delta_{p}(j=x, y, z)$. The free 


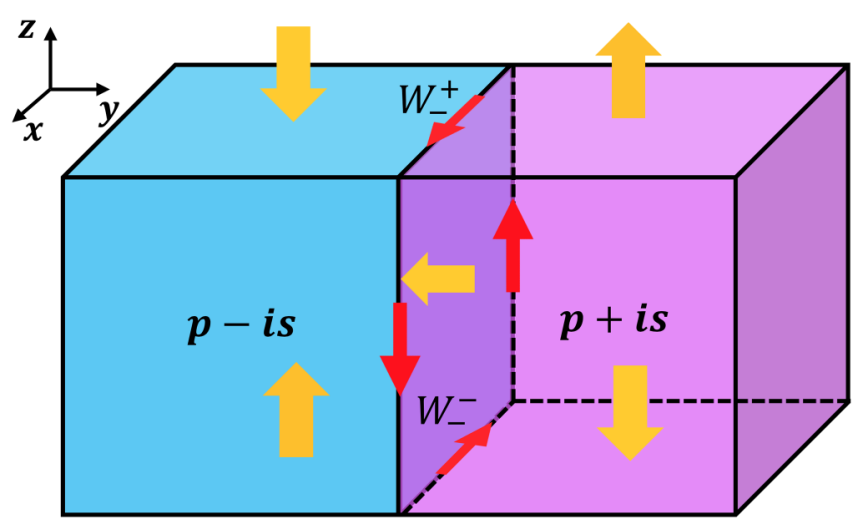

FIG. 1. The propagating chiral Majorana modes represented by red arrows. The yellow arrows in the figure represent the directions of magnetizations at several selected surfaces or interfaces.

energy acquires the following terms under $\vec{h}(\vec{r})$ as

$$
\begin{aligned}
\Delta F^{(3)}= & \frac{1}{3} D \epsilon_{f} \int d^{3} \vec{r} \vec{h} \cdot \operatorname{Im}\left[-\left(\nabla \Delta_{s}\right) \Delta_{p}^{*}+\Delta_{s} \nabla \Delta_{p}^{*}\right], \\
\Delta F^{(4)}= & D \int d^{3} \vec{r} \vec{h} \cdot \operatorname{Im}\left[(\nabla V) \Delta_{s} \Delta_{p}^{*}-V\left(\nabla \Delta_{s}\right) \Delta_{p}^{*}\right. \\
& \left.+V \Delta_{s} \nabla \Delta_{p}^{*}\right],
\end{aligned}
$$

in which $\epsilon_{f}$ is the Fermi energy; $k_{f}$ is the Fermi wave vector; and $D=N_{f} \frac{1}{k_{f}} \frac{7 \zeta(3)}{(8 \pi)^{2}} \frac{1}{T_{c}^{2}}$ where $N_{f}$ is the density of states at the Fermi energy, $T_{c}$ is the superconducting transition temperature, and $\zeta$ is the Riemann zeta function. The derivations of Eq. (10) are included in SM Sec. V [61]. Since the magnetization $\vec{M}$ is conjugate to $\vec{h}$, all $\nabla \Delta_{s}, \nabla \Delta_{p}$, and $\nabla V$ can induce $\vec{M}$.

The first term in $\Delta F^{(4)}$ leads to the magnetoelectric effect. The magnetization induced by the spatial variation of the external potential is given by $\vec{M}(\vec{r})=\chi \nabla V(\vec{r})$, where $\chi=D \operatorname{Im}\left(\Delta_{p} \Delta_{s}^{*}\right)$. A nonzero $\chi$ requires the coexistence of $\Delta_{s}$ and $\Delta_{p}$ with a phase difference not equal to 0 or $\pi$, hence, both the inversion and time-reversal symmetries are broken. As analyzed before, the $p \pm i$ s pairing gap functions are energetically favored when they are nearly degenerate. In this case, $\chi$ is nonzero and its sign is opposite for the $p \pm i$ s cases. The open boundary condition used previously corresponds to a sudden jump of the electric potential. Hence, the spin-polarized surface state is a manifestation of the magnetoelectric effect.

Magnetizations can also be induced by the spatial inhomogeneity of the superconducting gap functions as described in $\Delta F^{(3)}$. This effect is embodied in the spontaneous magnetization at the interfaces between regions with different pairing symmetries in the bulk as shown in Fig. 1(b). Furthermore, for the interface between the $p+i$ s and $p-i s$ regions, we find localized and spin-polarized midgap states at the energy of $\left|\Delta_{p}\right|$, while for the interface between the $p+i s$ and $-p+i$ s regions, the energies are at $\left|\Delta_{S}\right|$. The solutions of these midgap states are included in SM Sec. VI [61]. We also note that the chiral Majorana fermion can alternatively be viewed as propagating on the edge of such interfaces.
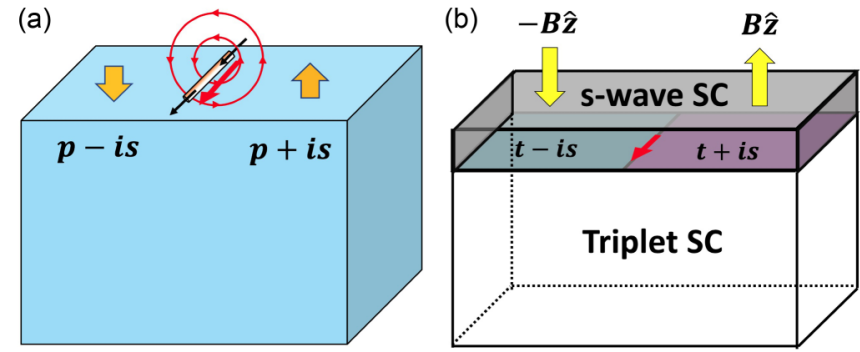

FIG. 2. The propagating chiral Majorana fermion (represented by the red straight arrows) produced by (a) a current-carrying wire placed on top of a $p \pm i$ s superconductor, and (b) a triplet pairing superconductor in proximity with an $s$-wave superconducting thin film. In (a), the black straight arrows, the red circled arrows, and the orange fat arrows represent the electrical currents flowing through the wire, the magnetic fields generated by the current, and the surface magnetizations, respectively. In (b), the yellow arrows represent the directions of the external magnetic fields.

The chiral Majorana fermion can be produced and controlled by a current-carrying wire placed on top of the surface of the system as shown in Fig. 2(a). The directions of the magnetic fields on the surface produced by the wire are antiparallel on the opposite sides of the wire, thus the induced symmetry breaking pattern $(p+i s$ or $p-i s)$ changes across the wire when the system is cooled below $T_{c}$. As discussed previously, there exists a chiral Majorana fermion propagating along the domain wall produced by the electric current. The domain wall will follow the motion of the wire if the motion is slow enough to ensure adiabaticity. Hence, such chiral Majorana fermion is mobile and can be conveniently dragged by translating the wire on the surface.

Besides the intrinsic $p \pm i$ s superconductivities, our strategy for realizing mobile chiral Majorana fermions can also be carried out using proximity effect. There is experimental evidence for the $\mathrm{Cu}_{x} \mathrm{Bi}_{2} \mathrm{Se}_{3}$ and $\mathrm{Sn}_{1-x} \mathrm{In}_{x} \mathrm{Te}$ materials to host a time-reversal invariant triplet superconductivity [36,38-40]. In these two materials, although the proposed triplet pairing symmetry ( $A_{1 u}$ representation of the $D_{3 \mathrm{~d}}$ point group) [35] is not the same as the $p$-wave pairing discussed in our work, $C_{\text {ch }}$ is still an antisymmetry (i.e., anticommuting with the Hamiltonian), and $S_{z}$ is in the little group of $\vec{k} \| \hat{z}$ since the $C_{3}$ symmetry is unbroken. Hence, the presence of an $s$-wave pairing will play exactly the same role as discussed in our work in splitting the Majorana zero modes and creating a spontaneous magnetization on the surface with the normal direction along $\hat{z}$. As shown in Fig. 2(b), by coating an $s$-wave superconducting thin film on top of the triplet superconducting bulk, the unitary $t \pm i$ is pairing symmetry ( $t$ for the above mentioned $A_{1 u}$ pairing) is energetically favored close to the interface between the $s$-wave film and the triplet pairing bulk due to the proximity effect. The phase difference between the singlet and triplet pairing components (i.e., $t+i s$ or $t-i s$ ) can be conveniently controlled by external magnetic fields, and there exists a chiral Majorana fermion propagating along the domain wall separating the $t+i s$ and the $t-i$ s regions on the interface.

In summary, we have proposed that both the localized Majorana zero-energy states and the dispersive chiral ones 
can be realized via the kink on boundary method. The boundaries of $p \pm i$ s superconductors are spontaneously magnetized, with opposite directions of magnetizations for the $p+i s$ and $p-i s$ pairings, as a manifestation of the magnetoelectric effect. Along the $1 \mathrm{D}$ domain wall between the $p \pm i$ s domains on the surface, there exists a chiral Ma- jorana mode propagating unidirectionally, which can be controlled by magnetic fields. Our discussions are relevant to superconducting materials with competing singlet and triplet pairing orders and proximity-effect-induced superconductivities with a mixed singlet and triplet pairing symmetry.
[1] E. Majorana, Teoria simmetrica dell'elettrone e del positrone, Nuovo Cimento 14, 171 (1937).

[2] C. Nayak, S. H. Simon, A. Stern, M. Freedman, and S. Das Sarma, Non-Abelian anyons and topological quantum computation, Rev. Mod. Phys. 80, 1083 (2008).

[3] G. Moore and N. Read, Nonabelions in the fractional quantum Hall effect, Nucl. Phys. B 360, 362 (1991).

[4] C. Nayak and F. Wilczek, $2 n$-quasihole states realize $2^{n-1}$ dimensional spinor braiding statistics in paired quantum Hall states, Nucl. Phys. B 479, 529 (1996).

[5] D. A. Ivanov, Non-Abelian Statistics of Half-Quantum Vortices in $p$-Wave Superconductors, Phys. Rev. Lett. 86, 268 (2001).

[6] A. Stern, F. von Oppen, and E. Mariani, Geometric phases and quantum entanglement as building blocks for non-Abelian quasiparticle statistics, Phys. Rev. B 70, 205338 (2004).

[7] A. Kitaev, Fault-tolerant quantum computation by anyons, Ann. Phys. 303, 2 (2003)

[8] A. Kitaev, Anyons in an exactly solved model and beyond, Ann. Phys. 321, 2 (2006).

[9] M. Stone and S.-B. Chung, Fusion rules and vortices in $p_{x}+i p_{y}$ superconductors, Phys. Rev. B 73, 014505 (2006).

[10] J. Alicea, Y. Oreg, G. Refael, F. von Oppen, and M. P. A. Fisher, Non-Abelian statistics and topological quantum information processing in 1D wire networks, Nat. Phys. 7, 412 (2011).

[11] B. I. Halperin, Y. Oreg, A. Stern, G. Refael, J. Alicea, and F. von Oppen, Adiabatic manipulations of Majorana fermions in a three-dimensional network of quantum wires, Phys. Rev. B 85, 144501 (2012).

[12] M. Z. Hasan and C. L. Kane, Colloquium: Topological insulators, Rev. Mod. Phys. 82, 3045 (2010).

[13] X.-L. Qi and S.-C. Zhang, Topological insulators and superconductors, Rev. Mod. Phys. 83, 1057 (2011).

[14] Y. Ando and L. Fu, Topological crystalline insulators and topological superconductors: From concepts to materials, Annu. Rev. Condens. Matter Phys. 6, 361 (2015).

[15] M. Sato and S. Fujimoto, Majorana fermions and topology in superconductors, J. Phys. Soc. Jpn. 85, 072001 (2016).

[16] C.-K. Chiu, J. C. Y. Teo, A. P. Schnyder, and S. Ryu, Classification of topological quantum matter with symmetries, Rev. Mod. Phys. 88, 035005 (2016).

[17] M. Sato and Y. Ando, Topological superconductors: A review, Rep. Prog. Phys. 80, 076501 (2017).

[18] A. Y. Kitaev, Unpaired Majorana fermions in quantum wires, Phys. Usp. 44, 131 (2001).

[19] N. Read and D. Green, Paired states of fermions in two dimensions with breaking of parity and time-reversal symmetries and the fractional quantum Hall effect, Phys. Rev. B 61, 10267 (2000).
[20] L. Fu and C. L. Kane, Superconducting Proximity Effect and Majorana Fermions at the Surface of a Topological Insulator, Phys. Rev. Lett. 100, 096407 (2008).

[21] J. D. Sau, R. M. Lutchyn, S. Tewari, and S. Das Sarma, Generic New Platform for Topological Quantum Computation using Semiconductor Heterostructures, Phys. Rev. Lett. 104, 040502 (2010).

[22] S. Das Sarma, C. Nayak, and S. Tewari, Proposal to stabilize and detect half-quantum vortices in strontium ruthenate thin films: Non-Abelian braiding statistics of vortices in a $p_{x}+i p_{y}$ superconductor, Phys. Rev. B 73, 220502(R) (2006).

[23] J. C. Y. Teo and C. L. Kane, Majorana Fermions and NonAbelian Statistics in Three Dimensions, Phys. Rev. Lett. 104, 046401 (2010).

[24] R. Yu, W. Zhang, H.-J. Zhang, S.-C. Zhang, X. Dai, and Z. Fang, Quantized anomalous Hall effect in magnetic topological insulators, Science 329, 61 (2010).

[25] X.-L. Qi, T. L. Hughes, and S.-C. Zhang, Chiral topological superconductor from the quantum Hall state, Phys. Rev. B 82, 184516 (2010).

[26] S. B. Chung, X.-L. Qi, J. Maciejko, and S.-C. Zhang, Conductance and noise signatures of Majorana backscattering, Phys. Rev. B 83, 100512(R) (2011).

[27] M. Cheng, R. M. Lutchyn, V. Galitski, and S. Das Sarma, Splitting of Majorana-Fermion Modes Due to Intervortex Tunneling in a $p_{x}+i p_{y}$ Superconductor, Phys. Rev. Lett. 103, 107001 (2009).

[28] Y. Li, D. Wang, and C. Wu, Spontaneous breaking of timereversal symmetry in the orbital channel for the boundary Majorana flat bands, New J. Phys. 15, 085002 (2013).

[29] A. C. Potter and P. A. Lee, Edge Ferromagnetism from Majorana Flat Bands: Application to Split Tunneling-Conductance Peaks in High- $T_{c}$ Cuprate Superconductors, Phys. Rev. Lett. 112, 117002 (2014).

[30] Y.-J. Doh, J. A. van Dam, A. L. Roest, E. P. A. M. Bakkers, L. P. Kouwenhoven, and S. D. Franceschi, Tunable supercurrent through semiconductor nanowires, Science 309, 272 (2005).

[31] H. H. Sun, K. W. Zhang, L. H. Hu, C. Li, G. Y. Wang, H. Y. Ma, Z. A. Xu, C. L. Gao, D. D. Guan, Y. Y. Li, C. Liu, D. Qian, Y. Zhou, L. Fu, S. C. Li, F. C. Zhang, and J. F. Jia, Majorana Zero Mode Detected with Spin Selective Andreev Reflection in the Vortex of a Topological Superconductor, Phys. Rev. Lett. 116, 257003 (2016).

[32] B. Jäck, Y. Xie, J. Li, Sangjun Jeon, B. Andrei Bernevig, and Ali. Yazdani, Observation of a Majorana zero mode in a topologically protected edge channel, Science 364, 1255 (2019).

[33] H. Sakai, K. Yoshimura, H. Ohno, H. Kato, S. Kambe, R. E. Walstedt, T. D. Matsuda, Y. Haga, and Y. Onuki, Superconductivity in a pyrochlore oxide, $\mathrm{Cd}_{2} \mathrm{Re}_{2} \mathrm{O}_{7}$, J. Phys.: Condens. Matter 13, L785 (2001). 
[34] C. Wu and J. E. Hirsch, Mixed triplet and singlet pairing in ultracold multicomponent fermion systems with dipolar interactions, Phys. Rev. B 81, 020508(R) (2010).

[35] L. Fu and E. Berg, Odd-Parity Topological Superconductors: Theory and Application to $\mathrm{Cu}_{x} \mathrm{Bi}_{2} \mathrm{Se}_{3}$, Phys. Rev. Lett. 105, 097001 (2010).

[36] S. Sasaki, M. Kriener, K. Segawa, K. Yada, Y. Tanaka, M. Sato, and Y. Ando, Topological Superconductivity in $\mathrm{Cu}_{x} \mathrm{Bi}_{2} \mathrm{Se}_{3}$, Phys. Rev. Lett. 107, 217001 (2011).

[37] T. C. Kobayashi, Y. Irie, J. ichi Yamaura, Z. Hiroi, and K. Murata, Superconductivity of heavy carriers in the pressureinduced phases of $\mathrm{Cd}_{2} \operatorname{Re}_{2} \mathrm{O}_{7}$, J. Phys. Soc. Jpn. 80, 023715 (2011).

[38] T. Kirzhner, E. Lahoud, K. B. Chaska, Z. Salman, and A. Kanigel, Point-contact spectroscopy of $\mathrm{Cu}_{0.2} \mathrm{Bi}_{2} \mathrm{Se}_{3}$ single crystals, Phys. Rev. B 86, 064517 (2012).

[39] S. Sasaki, Z. Ren, A. A. Taskin, K. Segawa, L. Fu, and Y. Ando, Odd-Parity Pairing and Topological Superconductivity in a Strongly Spin-Orbit Coupled Semiconductor, Phys. Rev. Lett. 109, 217004 (2012).

[40] M. Novak, S. Sasaki, M. Kriener, K. Segawa, and Y. Ando, Unusual nature of fully gapped superconductivity in In-doped SnTe, Phys. Rev. B 88, 140502(R) (2013).

[41] A. Hinojosa, R. M. Fernandes, and A. V. Chubukov, TimeReversal Symmetry Breaking Superconductivity in the Coexistence Phase with Magnetism in Fe Pnictides, Phys. Rev. Lett. 113, 167001 (2014).

[42] L. Zhou, W. Yi, and X. Cui, Fermion superfluid with hybridized $s$ - and $p$-wave pairings, Sci. China Phys. Mech. Astron. 60, 127011 (2017).

[43] Y. Wang and L. Fu, Topological Phase Transitions in Multicomponent Superconductors, Phys. Rev. Lett. 119, 187003 (2017).

[44] S. Ryu, J. E. Moore, and A. W. W. Ludwig, Electromagnetic and gravitational responses and anomalies in topological insulators and superconductors, Phys. Rev. B 85, 045104 (2012).

[45] X.-L. Qi, E. Witten, and S.-C. Zhang, Axion topological field theory of topological superconductors, Phys. Rev. B 87, 134519 (2013).

[46] K. Shiozaki and S. Fujimoto, Dynamical axion in topological superconductors and superfluids, Phys. Rev. B 89, 054506 (2014).

[47] P. Goswami and B. Roy, Axionic superconductivity in threedimensional doped narrow-gap semiconductors, Phys. Rev. B 90, 041301(R) (2014).

[48] P. Goswami and A. H. Nevidomskyy, Topological Weyl superconductor to diffusive thermal Hall metal crossover in the $B$ phase of $\mathrm{UPt}_{3}$, Phys. Rev. B 92, 214504 (2015).

[49] M. Stone and P. L. E. S. Lopes, Effective action and electromagnetic response of topological superconductors and Majorana-mass Weyl fermions, Phys. Rev. B 93, 174501 (2016).

[50] T. Shang, M. Smidman, S. K. Ghosh, C. Baines, L. J. Chang, D. J. Gawryluk, J. A. T. Barker, R. P. Singh, D. Mck. Paul, G. Balakrishnan, E. Pomjakushina, M. Shi, M. Medarde, A. D.
Hillier, H. Q. Yuan, J. Quintanilla, J. Mesot, and T. Shiroka, Time-Reversal Symmetry Breaking in Re-Based Superconductors, Phys. Rev. Lett. 121, 257002 (2018).

[51] S. Sundar, S. Salem-Sugui, Jr., M. K. Chattopadhyay, S. B. Roy, L. S. Sharath Chandra, L. F. Cohen, and L. Ghivelder, Study of $\mathrm{Nb}_{0.18} \operatorname{Re}_{0.82}$ non-centrosymmetric superconductor in the normal and superconducting states, Supercond. Sci. Technol. 32, 055003 (2019).

[52] W. A. Benalcazar, B. A. Bernevig, and T. L. Hughes, Quantized electric multipole insulators, Science 357, 61 (2017).

[53] Y. Wang, M. Lin, and T. L. Hughes, Weak-pairing higher order topological superconductors, Phys. Rev. B 98, 165144 (2018).

[54] X. Zhu, Second-Order Topological Superconductors with Mixed Pairing, Phys. Rev. Lett. 122, 236401 (2019).

[55] X.-H. Pan, K.-J. Yang, L. Chen, G. Xu, C.-X. Liu, and X. Liu, Lattice-Symmetry-Assisted Second-Order Topological Superconductors and Majorana Patterns, Phys. Rev. Lett. 123, 156801 (2019).

[56] Y. Volpez, D. Loss, and J. Klinovaja, Second-Order Topological Superconductivity in $\pi$-junction Rashba Layers, Phys. Rev. Lett. 122, 126402 (2019).

[57] N. Bultinck, B. A. Bernevig, and M. P. Zaletel, Threedimensional superconductors with hybrid higher-order topology, Phys. Rev. B 99, 125149 (2019).

[58] R.-X. Zhang, W. S. Cole, and S. Das Sarma, Helical Hinge Majorana Modes in Iron-Based Superconductors, Phys. Rev. Lett. 122, 187001 (2019).

[59] Z. Wu, Z. Yan, and W. Huang, Higher-order topological superconductivity: Possible realization in Fermi gases and $\mathrm{Sr}_{2} \mathrm{RuO}_{4}$, Phys. Rev. B 99, 020508(R) (2019).

[60] A. J. Leggett, A theoretical description of the new phases of liquid ${ }^{3} \mathrm{He}$, Rev. Mod. Phys. 47, 331 (1975).

[61] See Supplemental Material at http://link.aps.org/supplemental/ 10.1103/PhysRevResearch.2.042047 (which contains Ref. [65]) for detailed discussions about solutions of surface states on the boundary of $p \pm i$ s superconductors, numerical solutions of Majorana zero modes at the kink of boundary, symmetry operations, surface magnetization of $p \pm i$ s superconductors, calculations of the Ginzburg-Landau free-energy capturing the magnetoelectric effect, and gapped surface states at different interfaces.

[62] X. L. Qi, T. L. Hughes, S. Raghu, and S. C. Zhang, Time-Reversal-Invariant Topological Superconductors and Superfluids in Two and Three Dimensions, Phys. Rev. Lett. 102, 187001 (2009).

[63] A. P. Schnyder, S. Ryu, A. Furusaki, and A. W. W. Ludwig, Classification of topological insulators and superconductors in three spatial dimensions, Phys. Rev. B 78, 195125 (2008).

[64] X. L. Qi, T. Hughes, and S. C. Zhang, Fractional charge and quantized current in the quantum spin Hall state, Nat. Phys. 4, 273 (2008).

[65] D. H. Lee, Surface States of Topological Insulators: The Dirac Fermion in Curved Two-Dimensional Spaces, Phys. Rev. Lett. 103, 196804 (2009). 